\title{
La prééminence du document dans l'édition
}

\section{Hans-Walter Gabler}

\section{OpenEdition}

Journals

Édition électronique

URL : http://journals.openedition.org/recherchestravaux/85

DOI : $10.4000 /$ recherchestravaux.85

ISSN : 1969-6434

\section{Éditeur}

UGA Éditions/Université Grenoble Alpes

\section{Édition imprimée}

Date de publication : 15 juin 2008

Pagination : $39-51$

ISBN : 978-2-84310-125-0

ISSN : 0151-1874

\section{Référence électronique}

Hans-Walter Gabler, «La prééminence du document dans l'édition », Recherches \& Travaux [En ligne] 72 | 2008, mis en ligne le 15 décembre 2009, consulté le 03 février 2021. URL : http://

journals.openedition.org/recherchestravaux/85; DOI : https://doi.org/10.4000/recherchestravaux.85

(c) Recherches \& Travaux 
Hans-Walter GABLER*

Université de Munich

\section{La prééminence du document dans l'édition}

À la question posée jadis par Gunter Martens, spécialiste allemand de critique textuelle ${ }^{1}$ : «D'un point de vue éditorial, qu'est-ce qu'un texte? ?», je répondrai aujourd'hui par la question suivante: «D’un point de vue éditorial, qu'est-ce qu'un document?» Tant que la transmission et l'édition se trouvaient liées dans le même univers matériel d'encre et de papier, le problème ne se posait pas : le document était «transparent» parce qu'il était aussi matériel que tout ce qui l'entourait. Mais à l'heure où la virtualisation de l'édition savante progresse, la question se pose désormais et appelle une réponse. Traditionnellement, les documents portent le texte et en témoignent. Les documents d'une provenance auctoriale proche sont notamment considérés comme des garants d'auctorialité aussi bien que comme les référents matériels de telle ou telle version du texte. La tradition considère grosso modo les documents comme le substrat matériel de la transmission textuelle. Bien entendu, - si l'on excepte la transmission orale - il est vrai que sans documents il n'y aurait pas de textes. Pourtant, une généralisation inverse - si l'on excepte cette fois la pratique des collectionneurs de livres et de manuscrits - relègue aussitôt les documents au second plan que nos conventions leur assignent. Par

* Traduction de l'anglais par L. Garnier et C. Meynard, revue par Fr. Leriche et H.-W. Gabler.

I. Dans la tradition anglo-saxonne et germanique, la «critique textuelle» correspond à l'activité critique qui permet de recenser les transmissions, d'évaluer les documents et les variantes, et de définir les normes d'établissement de textes édités. Ce travail englobe l'activité éditoriale proprement dite, qui consiste à mettre en œuvre ces normes.

2. G. Martens, «Was ist - aus editorischer Sicht - ein Text? Überlegungen zur Bestimmung eines Zentralbegriffs der Editionsphilologie», Zu Werk, und Text. Beiträge zur Textologie, S. Scheibe et Chr. Laufer éd., Berlin, I 991, p. I 35 -I 56. 
conséquent, on peut dire tout aussi bien que notre intérêt culturel, et en particulier l'intérêt éditorial, pour la transmission a toujours privilégié le texte.

En ce qui concerne la transmission, les documents ont une double fonction. Ils donnent corps à tout ce qui est transmis mais, en tant que corps matériels, ils sont eux-mêmes périssables. Par conséquent, les pratiques culturelles de la transmission, et plus particulièrement celles de l'édition, tirent leur origine d'une prise de conscience de la mutabilité des documents et ont été inventées et développées afin de contrer leur dégradation et de leur perte. Mais cela signifie également que la transmission et l'édition ont toujours séparé le texte des documents : la transmission et l'édition d'un texte ne peuvent se faire qu'en l'extrayant d'un document pour le réinscrire sur un autre. Ainsi, l'édition traditionnelle laisse de côté les documents en tant que documents (on pourrait les jeter à la poubelle, les réutiliser pour la reliure de nouveaux volumes comme dans les bibliothèques du Moyen Âge, ou encore les fonds des bibliothèques pourraient être «désherbés», une fois le contenu des livres microfilmé ou numérisé). Même l'édition dite «documentaire» ne parvient pas dans les faits à éviter la transposition du texte d'un document vers un autre, quel que soit le niveau «diplomatique» imaginé pour coder les données du document premier, ou original.

Qui plus est, si l'on envisage la reproduction en fac-similé comme solution alternative à l'«édition diplomatique», n'est-il pas vrai que les éditions de facsimilés ne sont que rarement considérées comme des éditions? ou alors comme des éditions seulement dans la mesure où elles sont renforcées par des paratextes - parmi lesquels surtout des transcriptions? En effet, ces dernières nous permettent de lire ce qui, sur un fac-similé, peut seulement être $v u$. Une des raisons majeures qui expliquent la marginalisation de l'édition en facsimilé réside sans doute dans le fait que, précisément, elle n'extrait pas le texte du document. En fait, la marginalisation de l'édition en fac-similé me semble être un symptôme de la marginalisation voire de l'effacement du document originel dans l'édition traditionnelle, ou du moins de son ravalement au rôle auxiliaire de support du texte.

Or, les documents originaux ont des caractéristiques qu'aucune édition imaginable ne peut rendre de manière exhaustive ou qui, en vérité, ne se prêtent pas à l'édition. Un document original est un autographe et, par là même, ne peut être copié ou reproduit. En tant qu'original, il a le statut autographe d'une œuvre picturale (on pense notamment aux débats autour de La Joconde 3 ). À l'inverse, et pour reprendre le couple de termes rendu célèbre par Nelson Goodman, le document est un allographe si l'on considère son texte seul

3. D. Greetham, Textual Scholarship. An Introduction. New York, Garland, I 992. 
(Hamlet reste Hamlet, quel que soit le support de lecture ${ }^{4}$ ). En tant que document, il est unique : un document original est en fait unique à tous égards, sauf pour ce qui concerne l'intelligibilité de son texte.

Dans le medium matériel de papier et d'encre, l'édition du texte intelligible s'est développée comme un art de l'impossible : l'art de séparer effectivement les inséparables, à savoir le texte et son support matériel. Pour autant, cet art n'a été pratiqué, et n'a été praticable, qu'à une condition : la réinscription du texte, séparé de son support d'origine, sur un autre support matériel. À l'inverse, la réintroduction de l'image - par exemple, dans la reproduction en fac-similé - ne peut alors être comprise comme de l'«édition» au sens strict car, on l'a déjà dit, l'autographe en tant qu'autographe ne se prête pas à l'édition. Par conséquent, l'augmentation du nombre de reproductions en facsimilé dans les éditions imprimées, à laquelle on assiste depuis quelques années, pose un problème intéressant. De tels fac-similés s'élèvent rarement au-dessus d'un statut d'illustrations, mais quand c'est le cas, on accorde la prééminence au texte de l'édition (extrait du document même que l'on a reproduit en fac-similé) par rapport à la reproduction du document.

Or, même dans ce qui semble alors être une aporie, on arrive à discerner des possibilités qui, dans le medium matériel constitué par le papier et l'encre, n'ont pas été mises en œuvre - ou, en vérité, ne peuvent pas l'être. Le facsimilé donne à l'original une présence virtuelle. Cette dimension virtuelle est ce qu'une édition numérique peut mettre en œuvre de façon satisfaisante (et elle le fait déjà fréquemment) parce qu'elle met en œuvre cette virtualité de manière dynamique. Elle le peut parce que l'édition existe dans le medium électronique, c'est-à-dire parce que, avec l'avènement de l'édition électronique, il faut bien le reconnaitre, l'édition savante se fonde sur le transfert d'un medium vers un autre.

Mais cela peut - et à mon sens doit - mener à un questionnement plus profond et plus radical, et à de véritables reconceptualisations à la fois dans la théorie et dans la pratique. Je voudrais souligner à quel point il est nécessaire de nous interroger sur l'ordre canonique «texte et document», sur la pertinence qu'il y a à perpétuer cet ordre hiérarchique. Ce que je propose est une redéfinition de cette hiérarchie, qui en inverse l'ordre : inversion qui ne s'applique pas simplement à la pratique exploratoire de l'édition électronique mais, en définitive, à notre conceptualisation des «documents» et des «textes» dans tout le champ de la recherche universitaire et de la pensée critique. Désormais, il n'est plus nécessaire de définir le document comme fonction du

\footnotetext{
4. N. Goodman, Languages of Art. An Approach to a Theory of Symbols, Sussex, Harvester Press,
} 1968. 
texte, comme on le fait, implicitement ou explicitement, dans l'édition traditionnelle. Au contraire, il faudrait considérer le texte fondamentalement comme fonction du document - puisque, après tout, comme je l'ai dit initialement, ce sont des documents que nous avons, rien que des documents. Dans toute transmission et dans toute édition, les textes sont (et, sauf erreur, ont toujours été) des constructions à partir de documents C'est précisément cela que signifie éditer des textes de manière critique : les construire.

Ce bref aperçu théorique peut servir d'arrière-plan à une réflexion sur ma pratique actuelle. Depuis quelques années, je me suis engagé de plus en plus dans le champ de l'édition de manuscrits, et ce faisant, je me suis d'abord attaché à explorer les processus d'écriture dans les brouillons d'auteurs. Il est de la nature même de la pratique culturelle de l'écriture que les brouillons soient les documents de l'origine (et donc de l'inscription originelle) de tout texte survivant par la transmission - qu'ils survivent eux-mêmes ou non, en tant que sources originales de et pour l'écriture. Lorsque c'est le cas, ces documents peuvent susciter toutes sortes d'analyses, de questions et de problèmes fascinants. Une des questions, et en même temps un des problèmes, qui apparaissent à l'horizon de toute analyse aboutie d'un brouillon, est la suivante : peut-on éditer des brouillons (et non pas : peut-on éditer des textes à partir d'eux?) d'une manière, et avec des méthodes, qui rendent pleinement compte des indices des processus d'écriture et de pensée qui ont permis à ces brouillons de devenir les images (auto)graphiques que nous voyons en eux?

La pratique de la critique génétique peut nous guider pour suivre les processus de leur inscription et la dynamique de leur avènement. Généralement, cette pratique débouche tout d'abord sur un discours critique adapté de l'analyse des langues naturelles, qui sert à «réanimer les manuscrits dans leur temporalité» (comme l'a dit Almuth Grésillon lors du colloque ESTS en 2006 à Londres's). Généralement aussi, le discours critique s'appuie sur des restitutions représentationnelles de manuscrits par le biais de l'image, de la transcription et du codage métatextuel, c'est-à-dire par des restitutions qu'on appellerait conventionnellement «éditées». Cependant, et les généticiens seraient souvent les premiers à l'admettre, de telles représentations de manuscrits «éditées conventionnellement»sont, à l'encontre du discours critique qu'elles accompagnent, incapables de communiquer la dynamique et la temporalité de l'écriture manuscrite de manière immédiate, c'est-à-dire sans avoir recours aux abstractions du métadiscours éditorial que sont les sigles,

5. Le titre de son allocution au colloque était «Genetic Criticism, the Notion of avant-texte and the Question of Editing». 
les symboles, les différentiations typographiques ou les notes de bas de page. Cette limitation appelle au moins trois questions supplémentaires. Premièrement, à quel point la perception analytique de l'image d'une page manuscrite et de son existence dans la dynamique temporelle est-elle communicable? Deuxièmement, est-il possible, ou est-il même pertinent, de rechercher une approche éditoriale pour médiatiser cette perception? Et troisièmement, si c'est le cas, quels types d'approche critique pourraient prendre appui sur l'image d'une page manuscrite, à la suite d'une restitution éditoriale dynamique?

Mon postulat de départ, comme vous le savez sans doute, est celui de Daniel Ferrer qui a fait remarquer - et je l'ai déjà cité à maintes reprises - que «le brouillon n'est pas un texte ni un discours, c'est un protocole en vue de la fabrication d'un texte ${ }^{6} \%$. Ainsi, lorsque je regarde, par exemple, deux pages du brouillon (disjonctif) initial de James Joyce pour l'épisode de «Circé» dans Ulysse, je ne m'intéresse pas en premier lieu à la question de savoir quel texte les pages articulent. Je cherche plutôt à voir comment, en tant que pages, elles ont été successivement remplies (voir fig. I, infra, p. 44).

Cela revient à analyser les motifs d'encre et de crayon en ce qui concerne leurs caractéristiques d'inscription (analyse paléographique), leur positionnement absolu (analyse topographique) et leur positionnement relatif (analyse bibliographique). En eux-mêmes pourtant, ces relevés à la fois paléographiques, topographiques et bibliographiques, bien que nécessaires, ne sont pas suffisants. On ne peut, à terme, parvenir à établir l'ordre dans lequel les pages manuscrites ont été remplies qu'en procédant en même temps à une analyse qui discerne et distingue les unités d'écriture en les déchiffrant et en les lisant comme des segments de texte intelligible, c'est-à-dire en progressant de proche en proche, du document au texte, par des étapes de granularité de plus en plus fines. En d'autres termes, je pars du postulat que voir le document, analyser (et peut-être interpréter) les processus d'écriture selon lesquels il a été rempli, et lire (et peut-être interpréter) le texte qui, à terme, résulte de cette écriture, sont des activités bien distinctes; et que ces activités, en outre, doivent nécessairement se succéder dans cet ordre : du document, à l'écriture, et au texte. Certes, cela veut dire que, selon ma proposition théorique, je mets les bœufs du document bel et bien avant la charrue du texte qui finit par en émerger. Mais cela laisse encore ouverte la question de savoir s'il est possible

6. D. Ferrer, «The Open Space of the Draft Page : James Joyce and Modern Manuscripts», The Iconic Page in Manuscript, Print, and Digital Culture, Bornstein, G. et Th. Tinkle éd., Ann Arbor, University of Michigan Press, I998, p. 26r. La citation originelle est : «the draft is not a text, or a discourse; it is a protocol for making a text» (traduction de L. Garnier et C. Meynard). 


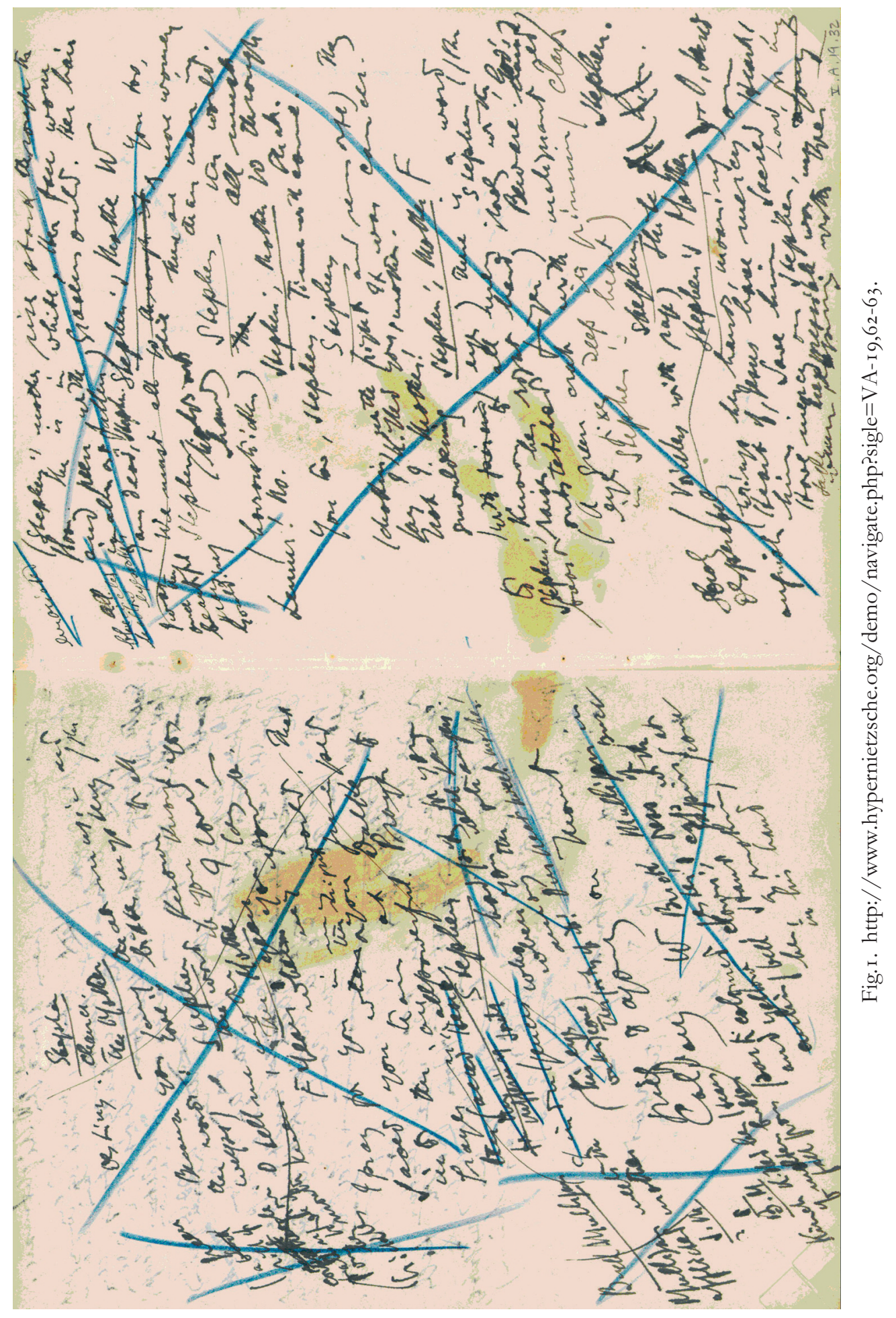


et intéressant, ou encore pertinent et gratifiant, d'explorer les modalités et les méthodes d'édition de l'écriture des manuscrits.

À l'origine de toute enquête, nous nous plaçons face à face avec la page (ou les pages) du manuscrit. Elles se présentent à nos yeux dans leur totalité, une totalité que nous pouvons percevoir, mais à laquelle nous ne pouvons initialement donner de sens (ne serait-ce que pendant une fraction de seconde ou pendant une période de temps considérable). En tentant alors d'aller au-delà de la surface de cette totalité, nous commençons à analyser et interpréter : à discerner, à distinguer, à lire. Pour une telle analyse critique, il nous faut l'image du manuscrit devant les yeux, que ce soit l'original ou toute sorte de reproduction. L'image numérisée affichée sur l'écran d'ordinateur ne constitue qu'un mode de reproduction parmi d'autres; l'ordinateur n'est pas, en tant que tel, essentiel pour nous permettre de voir et de «comprendre» la page manuscrite. Son pouvoir réside plutôt dans son potentiel de médiation : l'ordinateur nous invite à l'employer comme medium à la fois du processus éditorial et des éditions. En d'autres termes, bien plus radicaux : il nous invite à penser (et à continuer à penser) aux manuscrits et aux livres, aux textes et aux œuvres, comme solidement installés dans le royaume matériel du papier et de l'encre, tout en confiant au medium électronique et virtuel leur édition savante et la médiation éditoriale pour les présenter dans des éditions.

L'étendue et la complexité des possibilités de l'ordinateur, en tant que médiation et medium dans lequel l'édition savante existe, dépendent de l'étendue et de la complexité de notre apport éditorial. Pour ce qui est des images numérisées de pages manuscrites, j'imagine que leur qualité de présentation nous enchante tous. Mais l'intégration d'images de qualité supérieure n'est qu'un premier pas. Là où le medium électronique développe de plus en plus son potentiel, c'est dans les moyens qu'il offre pour enrichir le discours éditorial encadrant les images des manuscrits - un discours que nous reconnaissons alors aussitôt comme découlant fonctionnellement (ou dépendant autrement) des images numérisées d'abord intégrées dans le medium électronique. Par conséquent, nous créons un environnement de discours éditoriaux qui encadrent les images numérisées des manuscrits en consignant dans ce medium ce que nous avons analysé et interprété, discerné, distingué, et lu. En tant que discours éditoriaux, nos protocoles d'analyse peuvent prendre plusieurs formes, et souvent de façon simultanée.

Confronté à une image de manuscrit comme celle de la double page ouverte devant vous (fig. I), j'imagine que chacun mettrait en scène son analyse comme un dialogue dans lequel il avancerait en suivant les étapes du raisonnement logique. J'ai fait ailleurs la démonstration d'une telle analyse descriptive et tenté d'interpréter les sens et les significations des traces de l'écriture manuscrite de 
ces deux pages7. Je m'intéresserai ici aux manières dont, en premier lieu, je consigne dans le cadre virtuel qui contient l'image du manuscrit ce que j'ai observé et compris.

En travaillant entièrement sur la surface de cette image, je peux, dans un premier temps, la découper en la stratifiant en segments - et éventuellement mettre ces segments en relief avec des couleurs différentes. Cela donne une mise en page qui met d'abord en relief le fait que les deux pages ont été remplies par des inscriptions dans différentes zones topographiques, éparpillées de façon aléatoire sur l'espace de la double page. (Voir la démonstration sur le site : http://www.hypernietzsche.org/demo/navigate.php?sigle=VA-1 9,62-63.)

À l'étape suivante, je temporaliserais peut-être cette spatialisation, c'est-àdire que je donnerais dans mon compte rendu un aperçu de la dynamique du processus.

Si vous allez à l'adresse suivante : http://www.hypernietzsche.org/demo/ bksailehwgabler-I 7 , cliquez dans l'espace vide, et continuez de cliquer jusqu'à ce que la page soit remplie : ce que vous verrez est une simple suggestion de la façon dont une représentation virtuelle dynamique peut visualiser les processus temporels/spatiaux de l'écriture du manuscrit.

Ce que vous voyez est, en même temps, un protocole entièrement visuel du document, qui «processualise» l'image du manuscrit mais, pour l'instant, pas son inscription textuelle. Or, en face des manuscrits, c'est l'inscription que nous désirons pénétrer - et que, dans une certaine mesure, nous avons déjà pénétrée, afin même d'obtenir notre protocole visuel. Pour médiatiser la façon dont nous percevons l'inscription textuellement intelligible, nous allons changer de protocole : nous allons recourir non pas à une remise en image purement visuelle mais à la transcription.

Le protocole de transcription est majoritairement textuel; mais en même temps il dépend résolument du document. En clair, et selon l'ordre hiérarchique que je défends, le texte établi en transcription est une fonction du document. Ainsi, en tant que transcription, ce texte conserve - ou peut conserver selon la volonté du transcripteur - une dimension visuelle.

Comme vous le savez, Hyper ${ }^{8}$ a hérité du domaine de l'édition papier des conventions de transcription qui distinguent, sans surprise, transcription

7. Voir H.-W. Gabler, «For Ulysses: A Once and a Future Edition», Variants. The Journal of the European Society for Textual Scholarship, H. T. M. Van Vliet et P. M. W. Robinson éd., vol. I, 2002, p. 87-100.

8. Hyper est l'infrastructure numérique à laquelle j'ai recours dans mon propre travail pratique; voir M. Barbera, «Hyper. The Software Architecture», P. D’Iorio, «Nietzsche on New Paths», et H.-W. Gabler, «On HYPER». Tous ces documents sont téléchargeables en format PDF dans la rubrique «About the HyperNietzsche Project» sur la page d'accueil du site HyperNietzsche, http://www.hypernietzsche.org/. 
«diplomatique» et «transcription linéaire». L'application de ces conventions aux zones d'inscription/de transcription dans mon exemple de Joyce donne ceci :

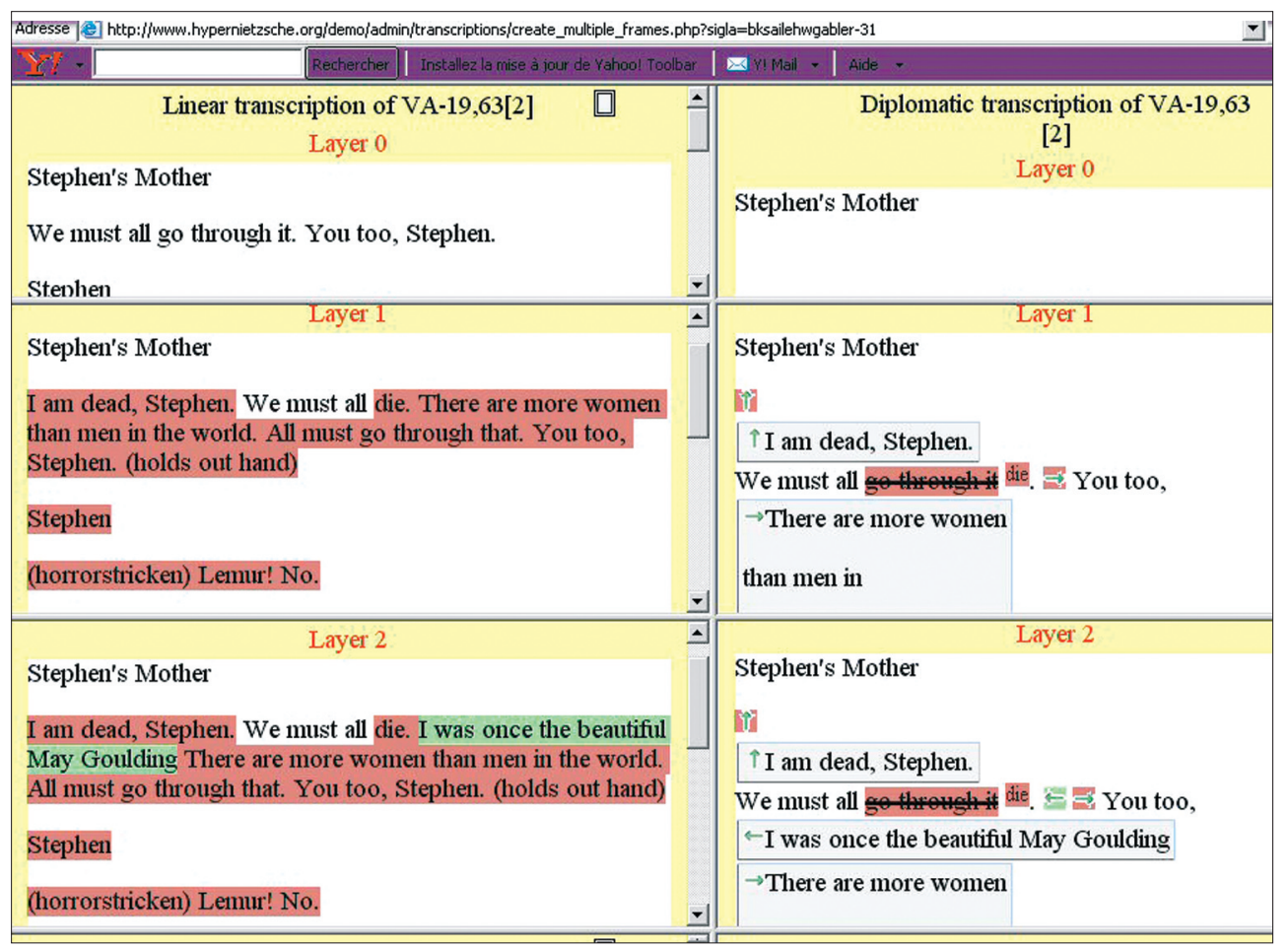

Fig. 2. http://www.hypernietzsche.org/demo/bksailehwgabler-3 I .

Le système fait apparaître en colonnes parallèles une transcription linéaire (à gauche) et une transcription diplomatique (à droite). La transcription linéaire représente le résultat (final) de l'écriture sur une certaine partie du manuscrit. La transcription diplomatique, à l'inverse, cherche à rendre le processus d'écriture du manuscrit. Ses lignes de texte superposées, ses cadres et ses flèches, sont des indications schématisées des niveaux et de la topographie des inscriptions dans le manuscrit. Cela devient directement perceptible si, à la place de la transcription linéaire, on fait apparaître l'image du manuscrit dans la partie gauche de l'affichage en deux colonnes (voir fig. 3 infra, p. 48).

Nous pouvons voir immédiatement, par confrontation avec l'image, ce que les dispositions de la transcription diplomatique sont conçues pour indiquer et nous voyons en même temps que, dans la transcription diplomatique, la représentation du manuscrit est schématisée. La référence visuelle à ce dernier est un élément important, mais il est clair que la transcription diplomatique 


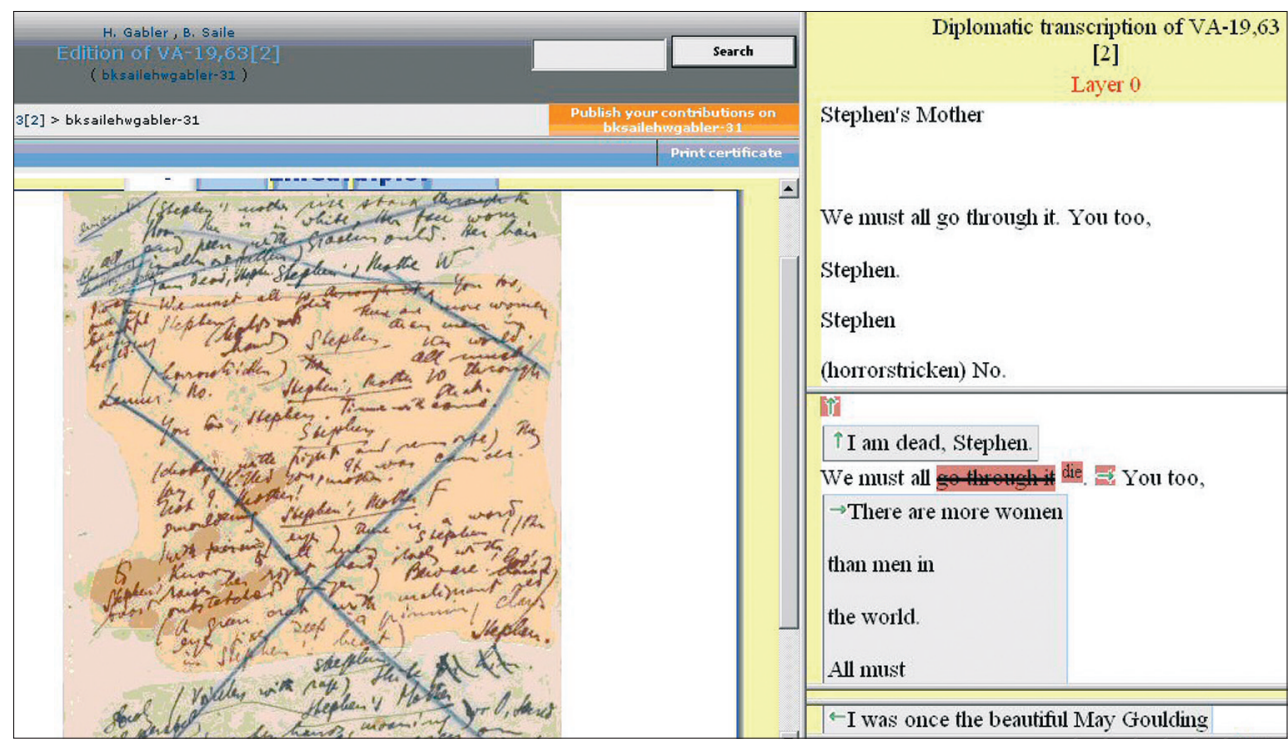

Fig. 3. L'image en regard de la transcription diplomatique.

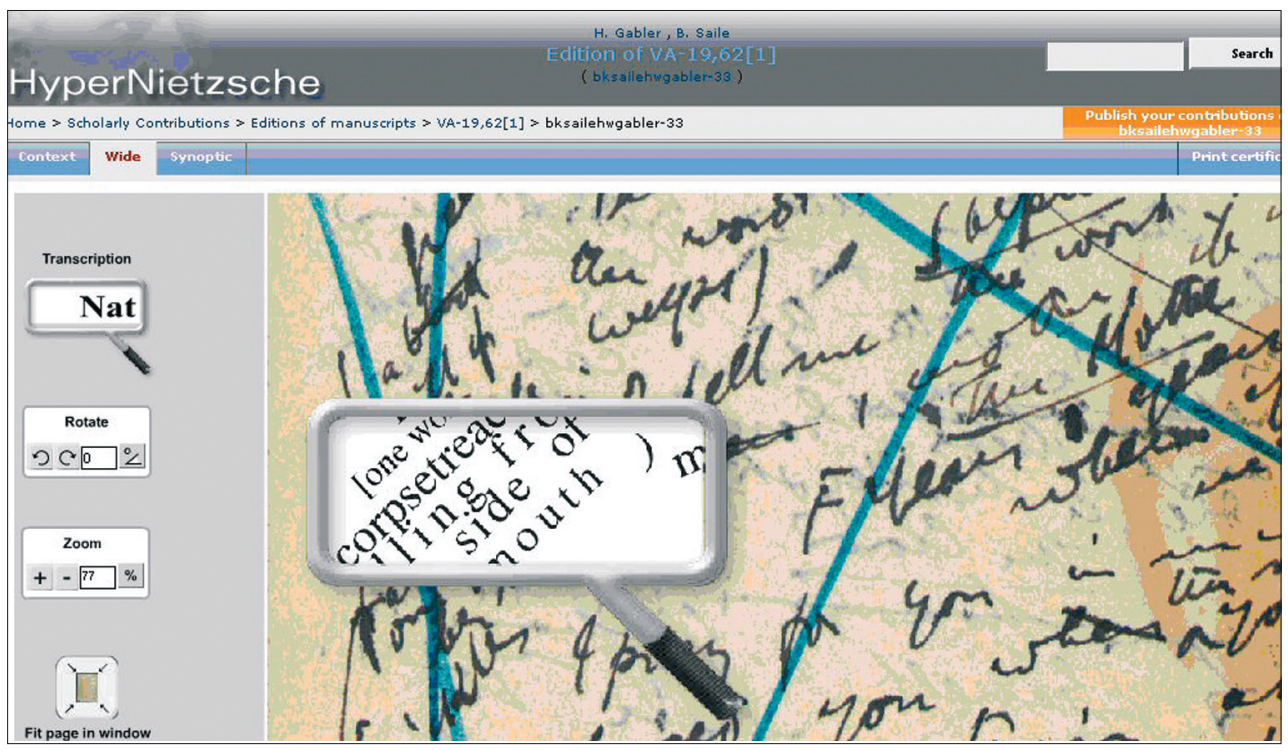

Fig. 4. La loupe permet d'accéder à une transcription «ultra-diplomatique» du brouillon (http://www.hypernietzsche.org/demo/bksailehwgabler-33). 
constitue déjà une abstraction distincte du document. Ce processus d'abstraction est évidemment poussé plus loin dans la transcription linéaire, qui n'exige plus - et ne nécessite plus - qu'on se reporte à l'image du manuscrit.

Autrement dit, le processus éditorial consistant à extraire le texte d'un original (et dans ce cas, de son substitut virtuel) et à le réinscrire commence déjà avec la transcription diplomatique. Or la coexistence, dans le medium électronique, de la forme visuelle du manuscrit (comme image) et de sa forme textuelle (dans une transcription diplomatique), nous permet de prendre en considération, les bénéfices que l'on peut retirer de l'association simultanée de ces types de consignation et de protocoles éditoriaux. C'est une direction qui a été prise, dans Hyper, avec la conception d'un mode de transcription qu'on appelle «ultra-diplomatique» (voir fig. 4 supra, p. 48).

Alors que la transcription diplomatique est encore la copie numérique d'un mode de présentation consacré dans le domaine du papier, seul le medium électronique peut permettre la transcription ultra-diplomatique. Voir à l'écran l'effet de la loupe sur l'image suffit à suggérer la capacité que possède le medium électronique pour démontrer l'étroite interdépendance de la visualisation et de la lecture d'un document. Dans la transcription ultra-diplomatique, l'interpénétration de l'image et du texte devient vraiment essentielle à la médiation éditoriale du document. Sous les auspices de la prééminence du document, on peut concevoir des avancées radicales dans l'édition de manuscrits proprement dite en sondant en profondeur l'interdépendance de l'image et de l'écriture au moyen de l'approche «ultra-diplomatique».

Enfin, j’ai indiqué plus haut que, à l'autre bout de l'échelle des transcriptions, la transcription «linéaire» a plus ou moins rompu ses liens avec le document. Cela semble nous ramener là où nous en étions à l'époque où il n'existait que l'édition imprimée sur papier, et où la transcription n'était que la première étape de l'édition du texte seul. Mais je ne veux pas tout à fait en rester là. De fait, les transcriptions linéaires dans le medium électronique (et en ce qui concerne les options fournies par l'infrastructure Hyper) ne présentent pas simplement le résultat (final) du processus d'écriture dans un document donné. De la même manière que sa transcription diplomatique peut être stratifiée en couches successives, de même, pour un document donné, on peut générer des transcriptions linéaires représentant successivement le résultat de l'écriture de ces différentes couches. La proposition de la «prééminence du document» prend alors une nouvelle dimension : à partir du document réel (c'est-à-dire ayant une existence matérielle) il est possible de générer des sousdocuments notionnels, et donc également virtuellement conçus, définis par les différents états de composition qu'on peut distinguer dans le processus de 
remplissage progressif du document réel. En effet, ce sera un des atouts formidables de l'édition électronique de manuscrits que de nous permettre de «déplier» les différents niveaux et étapes d'un processus de composition comme des textes intégraux séparés et distincts.

Concevoir un brouillon réel comme le télescopage et la superposition d'une série de sous-documents virtuels peut enfin nous amener à modéliser l'édition génétique à partir de plus d'un document ou, plus spécifiquement, d'une série consécutive de données matérielles sur la composition. Pour la représentation suivante, $j$ 'ai superposé les différents états textuels disponibles de la rencontre entre Stephen Dedalus et sa mère morte, la scène initialement esquissée dans le manuscrit qui a servi de point de départ à cette discussion.

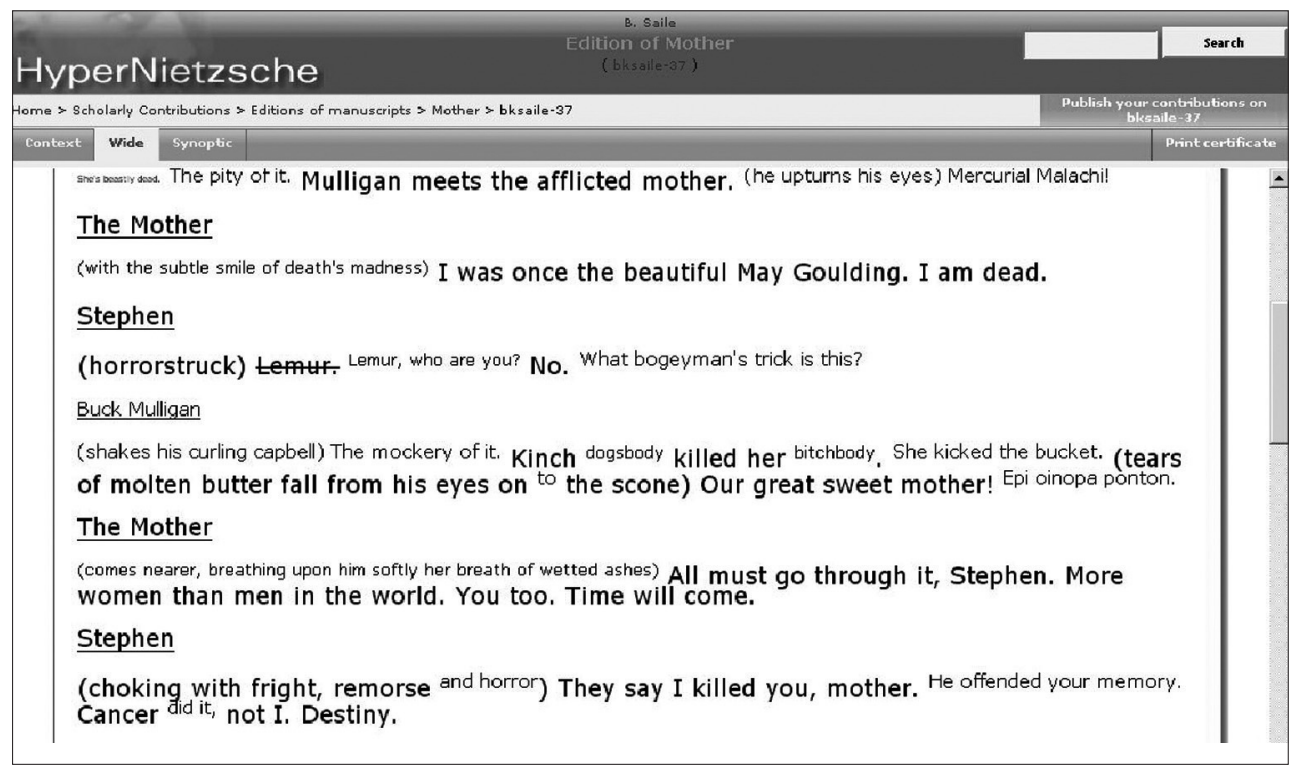

Fig. 5. Le document notionnel qui combine les états textuels d'une série de documents (http://www.hypernietzsche.org/demo/bksaile-37).

Les états textuels proviennent d'une série de documents allant du premier brouillon à la première édition, et je les ai combinés dans un seul fichier comme un seul ensemble de données. Naturellement, j'ai étiqueté l'état de chaque document individuel comme une strate de composition et de révision. Le programme informatique, de façon tout aussi évidente, gère chaque niveau d'étiquetage comme élément distinct dans une succession de strates inscrites comme sur un seul document. Ce document notionnel est le point focal virtuel de la genèse du texte qui s'étend, en réalité, sur une série de documents matériels. 
L'ordinateur, bien sûr, ne se préoccupe pas d'une quelconque distinction entre réalité matérielle et construction logique. Il ne peut être adapté que pour un document qui, dans ses termes, est de toute façon entièrement virtuel. Ainsi, construire un document virtuel composite à partir des données matérielles de documents réels, et lui accorder la prééminence logique en ce qui concerne la médiation électronique d'une construction éditoriale, assure la viabilité et le succès de l'entreprise d'édition génétique.

Mais je m'aperçois soudain que ce terrain m'est familier. Il y a environ vingt-cinq ans, j’ai justement défini un tel document logique pour l'Ulysse de James Joyce ${ }^{9}$ afin de me concentrer sur le développement textuel de la composition du roman. Le texte de Joyce ${ }^{9}$ m'a semblé circuler librement au-dessus des - et entre les - documents sur lesquels il s'appuyait. Je l'ai défini comme un «texte manuscrit continu», que j'ai déployé comme la copie à partir de laquelle construire notre édition du roman de Joyce. Réinscrire ce texte manuscrit continu dans un document virtuel ou logique (intitulé par facilité le «manuscrit continu») était simplement un appui heuristique pour notre procédure textuelle (op. cit., vol. III, p. I 894- I 896 et I90 I). Document virtuel ou pas, c'était de toute évidence - concernant le raisonnement global - une fonction du texte du roman émergeant dans la composition. Aujourd'hui, plaidant pour la prééminence du document, j'aurais tendance à introduire une nouvelle rationalité dans cette édition. Commençant par le «manuscrit continu», je lui accorderais la prééminence globale, regroupant sous la prééminence du document virtuel celles de tous les documents existant matériellement. Le texte tel qu'il est présenté dans notre édition, à la fois en tant que texte génétiquement stratifié et comme édition critique d'un texte à lire, serait alors compris comme un ensemble de fonctions du document dérivant intégralement du manuscrit continu que j'ai défini, et le restituant sous des formes multiples.

En ce qui concerne l'édition d'Ulysse, il ne s'agit, bien sûr, que de quelques remarques provisoires à caractère auto-historicisant. En revanche, il faudra encore beaucoup de réflexion et de développement pratique pour connaittre les conséquences qui peuvent découler des différentes considérations que je viens de vous livrer au sujet des documents et des textes, de leur relation et de certains de leurs modes de présentation et de représentation dans le medium électronique de l'avenir.

9. J. Joyce, Ulysses. A Critical and Synoptic Edition. H.-W. Gabler, W. Steppe et Cl. Melchior éd., New York, Garland, i 984, 3 volumes. 